\title{
The MSc project in orthodontics - an evidence-based opportunity?
}

\section{Julian O'Neill}

Consultant Orthodontist, Kettering, Northamptonshire, UK

\begin{abstract}
In the UK, all students undergoing postgraduate training in orthodontics carry out an MSc project. Individual prospective clinical studies involving active therapy can rarely be completed in the short time available. The time allowed for an MSc project would, however, be ample to carry out a systematic review. This kind of project would also fulfil university regulations, which require the production of a piece of original research.
\end{abstract}

For any practitioner in dentistry, there is an awareness that professional knowledge diminishes with time and that new evidence does not arrive automatically in our surgeries and has to be sought out. Keeping abreast of new developments and accessing the evidence in journals can be very time-consuming; indeed, the increasing number of publications in dentistry and concurrent improvement in the quality of research papers makes the appraisal of all new literature bewildering.

A well-conducted systematic review of the literature, in any area of dental research, is a most useful tool for assessing, summarising and communicating the results and implications of otherwise unmanageable quantities of research. Systematic reviews must be carried out, as with any reputable piece of scientific research, using a methodology that is logical, well-described and repeatable. This is a difficult task requiring access to good library and search facilities.

To date, only a limited number of systematic reviews in dentistry have been completed. The Cochrane Oral Health Group website displays three completed reviews in dentistry (one in orthodontics), ${ }^{1}$ and lists 14 protocols as accepted, indicating systematic reviews in progress (two of which are about orthodontics). The NHS Centre for Reviews and Dissemination (a sibling organisation of the UK Cochrane Centre, established in 1994) also records five systematic reviews in dentistry, one per year in 1997, 1998 and 1999 and two in 2000 (none of which includes an orthodontic subject).

In orthodontics, all students undergoing postgraduate training carry out an MSc project. Many MSc courses are of 2 years' duration. Meanwhile, most active orthodontic treatment takes at least 18 months to complete. Postgraduate students need time to set up an experimental MSc project; individual prospective clinical studies involving active therapy are therefore rarely completed within the short time available. For those wishing to pursue the gold standard and carry out a prospective randomised controlled clinical trial (RCCT), even more time is required for ethical approval before commencing patient recruitment to such a study. The 2-year timeframe available to most postgraduate orthodontic specialist registrars is therefore suited only to projects that can be completed well within that period, allowing time for initial preparation, pilot study, the trial itself, data analysis, word processing and binding the finished product. Many projects are therefore either in vitro experiments using new orthodontic materials, human or animal tissue studies, retrospective study-cast and cephalometric analyses, or work based on questionnaires. For example, an analysis of MSc projects submitted at the Eastman Dental Institute, London, UK, in September 2000 yielded:

- six laboratory-based projects (three cell biology and three materialbased)

- three retrospective analyses of treatment outcomes

- one retrospective questionnaire dealing with treatment outcome

- one analysis of morphological features in two matched groups

- one analysis of an RCCT of two orthodontic treatment techniques (NP Hunt, personal communication, 2001). (This latter study was unusual, being possible only because the researcher was undertaking a 4year part-time MSc (2000).)

The time available for an MSc project would, however, be ample for carrying out a systematic review. This kind of project would also fulfil University regulations, which require the production of a piece of original research.

There may be a further advantage to the systematic review as part of the MSc. The time required to supervise a student carrying out a systematic review may be considerably less than that needed to oversee an experimental 
project: supervisor time in a busy academic orthodontic departments is at a premium. Systematic reviews as MSc projects could reduce this workload.

In addition to MSc students, postCCST (Certificate of Completion of Specialist Training) orthodontists undergoing further training in a 'FixedTerm Training Appointment' - who must carry out a further research project in partial fulfilment of the Intercollegiate Specialist Examination (ISE) regulations - would be in a good position to undertake a systematic review, particularly if their original MSc project was experimental in nature. This concept is already acceptable to the ISE board. It might therefore be hoped that more systematic reviews in orthodontics would result, although, to prevent duplication of effort, it would be necessary for any prospective systematic reviewer to know if similar work was already being undertaken elsewhere. Ideally, therefore, any reviewer should register their intention to proceed with a particular topic by submitting a protocol to a central register. In the UK there are a few possible sites for registering reviews in Medicine and Dentistry. These include the Centre for Evidencebased Dentistry, the Oral Health Group in the Cochrane Centre, or perhaps the NHS Centre for Reviews and Dissemination. This would allow potential reviewers to confirm the need for an update in their field of interest.

There is now a precedent for carrying out a systematic review as an MSc project. A review of the literature related to methods used to move maxillary molars distally was recently completed (G Atherton, personal communication, 2001). Not only were the findings reported to be very relevant, but they also had a direct influence on the reviewer's clinical practice. There is little doubt that an increasing number of systematic reviews of this kind in orthodontics, and indeed other areas in dentistry, will allow practitioners access to digestible synopses of relevant research and aid evidence-based decision making for their patients.

1. Harrison JE, Ashby D. Orthodontic treatments for posterior crossbites. Oral Health Module, Cochrane Library, 1999. 